\title{
Embryos and biopsies on the ChIP-ing forecast
}

UK researchers have devised a method
of chromatin immunoprecipitation that
requires as little as 100 cells, opening the
door to epigenetics studies in primary cells
and embryos.
Chromatin immunoprecipitation, ChIP, has been the workhorse of epigenetic studies for the past ten years. This technique allows researchers to determine which histone modifications are present at a given genomic location in the cell nucleus. Its principle is simple: while preserving the association of histones and DNA, one cuts the chromatin into small sections and precipitates it using antibodies against a given histone mark (a post-translational modification of an histone tail). The coprecipitated DNA can then be analyzed by PCR or hybridization.

Despite the technique's popularity, one of its drawbacks is the need for a large amount of starting material, typically ten million cells. "It is a recognized problem with ChIP that you are very restricted: you can work with cell lines but generally not with primary cells," says Bryan Turner of the University of Birmingham Medical School. A couple of years ago, frustrated by this problem, Turner and long-time colleague Laura O'Neill set out to find a solution. The main issue, they speculated, would be to optimize the efficiency of the procedure at every step. Preventing material loss and obtaining good-quality chromatin would be crucial. So they tried to 'bulk up' a small mouse chromatin sample using chromatin from another species as a carrier; thus, carrier ChIP was born (Fig. 1).

"It is a very simple concept that works extremely well," says O'Neill who, using fly SL2 cells as a source of carrier chromatin, rapidly succeeded in decreasing the required amount of starting material down to 10,000 then 1,000 mouse cells. Why stop there? Encouraged by the rapid success of the procedure, the pair was tempted to ask bolder and more challenging questions. And with the presence in the laboratory of embryologist graduate student Matthew VerMilyea, the challenge was obvious: could they look at histone marks in early embryos?

"It took at least a year," Turner recalls, "because we were going for a very small number of cells, much smaller than we had ever planned to try." In the July issue of Nature Genetics, the group reports how they have been able to make the procedure work reliably on as little as 50-100 cells, the pooled inner cell mass of three embryos. They looked at the distribution of histone marks such as $\mathrm{H} 3 \mathrm{~K} 9$ methylation and $\mathrm{H} 4$ acetylation on key regulator genes such as Nanog, Oct4 and Cdx2 in mouse stem cells. They observed striking differences between embryos' inner cell mass and embryonic stem cell lines, a result that is perhaps not surprising — as the epigenetic program is likely to have been altered during the adaptation of cells in culture-but serves as a cautionary tale for epigeneticists.

One of the keys to the success of this technical feat was the use of 'native' ChIP (NChIP; a protocol that O'Neill and Turner helped pioneer in the mid-1990s) instead of the more widespread XChIP, in which proteins are chemically cross-linked to DNA before the immunoprecipitation. Chemical cross-linking is necessary to look at nonhistone proteins, which otherwise detach from the DNA during the procedure, but it tends to affect the recognition of epitopes on the flexible histone tails, thus decreasing the efficiency of immunoprecipitation of histones. "Only a few people use NChIP," says Turner, "but there is no excuse for using any other technique if you are looking at histones because it is a very efficient procedure."

Of course there is a lot of interest in being able to look at non-histone proteins, and the Birmingham group is busy setting up the carrier ChIP procedure with cross-linking. O'Neill is optimistic about the outcome, "It will work but with a larger number of cells - 10,000 cells or perhaps $1,000-$ which is still several orders of magnitude less than what you need with conventional ChIP and still in the range of what you can

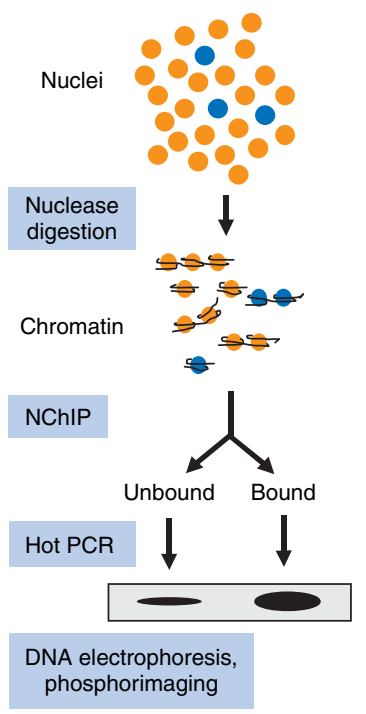

Figure 1 | Bulking up chromatin from mouse embryos (blue) using fly chromatin as 'carrier' (orange), Turner's group was able to perform ChIP on as little as 50 to 100 mouse cells, using the principle of native chromatin IP (NChIP). Reprinted from Nature Genetics, with permission.

obtain from a FACS sorted cell population or a biopsy."

The beauty of the system is that this new technique is giving ChIP the clinical relevance this classic was missing. Now chromatin immunoprecipitation can be envisioned on small sorted populations of immune cells or on cancer biopsies taken directly from patients. But what seems to excite Turner's group the most is really the embryo work. As Turner puts it, "The early embryo is the classic epigenetic system, that is where everything starts, and so far there has been no way to look at epigenetic marks in the early embryo." With this new technique, they may very well have opened a whole new field of study.

\section{Veronique Kiermer}

\section{RESEARCH PAPERS}

O'Neill L.P., VerMilyea M.D. \& Turner B.M. Epigenetic characterization of the early embryo with a chromatin immunoprecipitation protocol applicable to small cell populations. Nat. Genet. 38, 835-841 (2006). 